\title{
More on extending automorphisms of models of Peano Arithmetic
}

\author{
by \\ Roman Kossak (New York) and Henryk Kotlarski (Warszawa)
}

\begin{abstract}
Continuing the earlier research [Fund. Math. 129 (1988) and 149 (1996)] we give some information about extending automorphisms of models of PA to cofinal extensions.
\end{abstract}

In recent work on automorphisms of recursively saturated models of PA two themes stand out. One is the classification of conjugacy classes of single automorphisms, the other is the classification of subgroups of the automorphism group. Many results in both areas depend on information on how automorphisms of a recursively saturated model can be extended from the model to an elementary extension.

Let $\mathcal{M}$ be a countable model of Peano Arithmetic, PA, and let $\mathcal{N}$ be its cofinal extension. If $f$ is an automorphism of $\mathcal{M}, g$ is an automorphism of $\mathcal{N}$ and $f \subseteq g$, then for each $a \in \mathcal{N}$, there is $b \in \mathcal{N}$ such that $f(\mathcal{M} \cap a)=$ $\mathcal{M} \cap b$. This $b$ is, of course, $g(a)$. Here and elsewhere in the paper we identify elements of models of PA with the sets they code. If for each $a \in \mathcal{N}$, there is $b \in \mathcal{N}$ such that $f(\mathcal{M} \cap a)=\mathcal{M} \cap b$, we say that $f$ sends coded sets to coded sets. Thus, if $f$ has an extension to an automorphism of $\mathcal{N}$, then both $f$ and $f^{-1}$ send coded sets to coded ones. We showed in [9] that if $\mathcal{M}$ and $\mathcal{N}$ are countable and recursively saturated, $\mathcal{N}$ is an end elementary extension of $\mathcal{M}, \mathcal{M} \neq \inf \left\{(a)_{n}: n<\omega\right\}$ for all $a \in \mathcal{N}$, and $f$ is an automorphism of $\mathcal{M}$ such that $f$ and $f^{-1}$ send coded sets to coded ones, then $f$ can be extended to an automorphism of $\mathcal{M}$. In [10] we showed that the restriction on the type of end extension in the above result is essential. This, more or less, settles the problem of extending automorphisms of recursively saturated models to elementary end extensions. In [10] we considered extendability of automorphisms to cofinal extensions. This is a harder problem.

2000 Mathematics Subject Classification: 03C62, 03C50.

Key words and phrases: models of Peano Arithmetic, cofinal extensions, automorphisms. 
In the introductory Section 1 we give examples of automorphisms that do not extend to cofinal extensions, and we include two observations concerning extendability of automorphisms to simple cofinal extensions. Our main result appears in Section 2. We say that the extension $\mathcal{M} \prec_{\text {cof }} \mathcal{N}$ has the automorphism extension property if every automorphism $f$ of $\mathcal{M}$ such that $f$ and $f^{-1}$ send coded sets to coded ones can be extended to an automorphism of $\mathcal{N}$. We defined some other properties of cofinal extensions and showed that one of them implies the automorphism extension property. Here we give a shorter proof that a weaker property, which we call the description property (also defined in [10]), implies the automorphism extension property. In Section 3 we discuss some restrictions on the possible isomorphism types of structures of the form $(\mathcal{N}, \mathcal{M})$ where $\mathcal{M}$ is recursively saturated and $\mathcal{M} \prec_{\text {cof }} \mathcal{N}$. In Section 4 we consider a more refined extendibility question: Suppose $\mathcal{M}$ and $\mathcal{N}$ are countable and recursively saturated, $\mathcal{M} \prec \mathcal{N}$ and $f$ is an automorphism of $\mathcal{M}$ which can be extended to an automorphism of $\mathcal{N}$; can $f$ be extended to an automorphism $g$ such that $g$ moves all points in $\mathcal{N} \backslash \mathcal{M}$ ? We give an answer to this question in the case when $\mathcal{N}$ is an elementary end extension of $\mathcal{M}$. The result generalizes a theorem from [8], which is a special case for $f=\mathrm{id}$.

1. Automorphisms that do not extend. It is easy to see that if $f$ is a nontrivial automorphism of a model $\mathcal{M}$, then $\mathcal{M}$ has a cofinal extension to which $f$ does not extend. Here is the argument: Suppose $a<f(a)$. We can assume that for all $n \in \mathbb{N}$, $\exp (n, a)<f(a)$, where $\exp (n, x)$ is $2^{x}$ iterated $n$ times. If $a$ is not like that already we can consider $a^{\prime}=F(a)$ instead, where $F(x)$ is a definable function such that for all nonstandard $a$ and all $\Sigma_{1}$ definable $h(x, y)$, and all $x<a, \mathcal{M} \models F(a)>h(x, a)$ (see [14] for the construction of such an $F$ ). If $a$ is as above, then let $I$ be a cut of $\mathcal{M}$ which is closed under exponentiation and such that $a \in I<f(a)$. By a result of Smoryński [17] there exists $\mathcal{N} \succ \mathcal{M}$ such that $I$ is in the greatest common initial segment of $\mathcal{M}$ and $\mathcal{N}$. Then $f$ cannot be extended to $\mathcal{N}$. If $g$ were such an extension, then for any $b \in \mathcal{N} \backslash \mathcal{M}$ such that $b<f(a)$, we have $g^{-1}(b)<a$ and $g^{-1}(b) \notin M$, which is impossible.

The argument above shows how to kill a single automorphism. Now we will show how to kill continuum many. To do this we will use Schmerl's indiscernibles. Jim Schmerl proved in [15] that every countable recursively saturated model $\mathcal{M}$ is generated by a set $X$ of (order) indiscernibles. The set $X$ can be chosen to be of any countable order type, as long as it has no last element. Moreover, if $Y \subseteq X$ has no last element, then the submodel of $\mathcal{M}$ generated by $Y$, which we denote by $\operatorname{Hull}(Y)$, is isomorphic to $\mathcal{M}$. See Section 5.5 in [13] for the proof of this result. 
THEOREM 1. Let $\mathcal{M}$ be a countable recursively saturated model of PA. Then there exists a cofinal extension $\mathcal{N}$ of $\mathcal{M}$ such that continuum many automorphisms of $\mathcal{M}$ cannot be extended to $\mathcal{N}$.

Proof. Let $X$ be a generating set of Schmerl indiscernibles for $\mathcal{M}$ of order type $(\mathbb{Q},<)$. Let $Y$ be a subset of $X$ of order type $(\mathbb{N},<)$ which is not cofinal in $X$. Since every order-preserving permutation of $X$ extends to an automorphism of $\mathcal{M}$, the set $\{f(Y): f \in \operatorname{Aut}(\mathcal{M})\}$ is of power continuum. Let $\mathcal{N}$ be a cofinal countable extension of $\mathcal{M}$ in which $Y$ is coded. Clearly, $\{f(Y): f \in \operatorname{Aut}(\mathcal{N})\}$ is countable. Hence, continuum many automorphisms of $\mathcal{M}$ cannot be extended to $\mathcal{N}$.

Let $\mathcal{M}, \mathcal{N}, X$ and $Y$ be as in the proof of Theorem 1, and let $\mathcal{K}=$ $\operatorname{Hull}^{\mathcal{N}}(Y)$. Since every infinite subset of $X$ without a last element generates a submodel of $M$ which is isomorphic to $\mathcal{M}$, every type realized in $\mathcal{M}$ can be realized by an element in $\mathcal{K}$ which is of the form $t(\bar{y})$, where $t$ is a Skolem term and $\bar{y}$ is a tuple of elements of $Y$. Now, if $Y$ is coded by an $a \in \mathcal{N}$, it follows from a lemma due to Ehrenfeucht and Gaifman [1], [4] (for the proof see also Theorem 1.7.2 in [13]) that $\operatorname{tp}(a)$ cannot be realized in $\mathcal{M}$. Hence, $\operatorname{SSy}(\mathcal{M})$ is a proper subset of $\operatorname{SSy}(\mathcal{N})$. It is open whether Theorem 1 can be improved to obtain $\operatorname{SSy}(\mathcal{M})=\operatorname{SSy}(\mathcal{N})$.

Let us close this section with two simple observations on automorphism that do extend.

Proposition 2. Let $\mathcal{M} \models \mathrm{PA}$, let $\mathcal{N}$ be a simple (i.e. generated by one element over $\mathcal{M})$ elementary extension of $\mathcal{M}$ and let $g \in \operatorname{Aut}(\mathcal{M})$. Then $g$ has at most one extension to an automorphism of $\mathcal{N}$.

Proof. Assume that $h, f \in \operatorname{Aut}(\mathcal{N})$ extend $g$. Then $h \circ f^{-1} \mid \mathcal{M}=$ id. In order to show that $h \circ f^{-1}=$ id it suffices to show that $e=h \circ f^{-1}(d)=d$, where $d$ denotes the generator of the extension. But for all formulas $\varphi$ with parameters $m \in \mathcal{M}$ we must have $\mathcal{N} \models \varphi(m, d) \equiv \varphi\left(h \circ f^{-1} m, e\right)$, so the result follows from the Ehrenfeucht-Gaifman lemma.

Proposition 3. Let $\mathcal{M}=\mathrm{PA}$ and let $\mathcal{N}$ be a simple elementary extension of $\mathcal{M}$ generated by an element d over $\mathcal{M}$. Let $g \in \operatorname{Aut}(\mathcal{M})$ be such that for some $a \in \mathcal{M}$ with $\mathcal{N} \models d<a, g \uparrow(<a)=\mathrm{id}$. Then $g$ extends to some $\hat{g} \in \operatorname{Aut}(\mathcal{N})$.

Proof. We assert that for every formula $\varphi$ and every $m \in \mathcal{M}, \mathcal{N} \models$ $\varphi(m, d) \equiv \varphi(g(m), d)$. Granted this we let $\hat{g}(t(m, d))=t(g(m), d)$ and easily check that this $\hat{g}$ is well defined on $\mathcal{N}$ and in fact it is an automorphism of $\mathcal{N}$. In order to check the above assertion we let $n=g(m)$ and see that for every formula $\varphi$ and every $m \in \mathcal{M}, \mathcal{M}=\forall x<a[\varphi(m, x) \equiv \varphi(n, x)]$ because $g(x)=x$. It follows that the same statement holds in $\mathcal{N}$, so it holds for $x=d$, so the assertion is verified. 
Observe that $\hat{g}(d)=d$ in the proof given above. Of course, under the assumption of this proposition this must be so because $\hat{g}$ is unique by Proposition 2 .

This is about all we know about extending automorphisms to simple cofinal extensions. The difficulty here is that one must have a candidate for $g(d)$, where $d$ is the generator of the extension. It must be of the form $t(m, d)$ for some Skolem term $t$ and some $m \in \mathcal{M}$, but it is unclear how to find it when $g \in \operatorname{Aut}(\mathcal{M})$ is given.

2. The description property. We say that the extension $\mathcal{M} \prec_{\text {cof }} \mathcal{N}$ has the description property if for every $a \in \mathcal{N} \backslash M$ there is a sequence $\left\langle Y_{n}: n \in \mathbb{N}\right\rangle$ which is coded in $\mathcal{N}$ and such that for all $n \in \mathbb{N}$ :

1. $Y_{n} \in \mathcal{M}$;

2. $\mathcal{M}=Y_{n+1} \subseteq Y_{n}$;

3. $\mathcal{N} \models a \in Y_{n}$;

4. for every set $X \in \mathcal{M}$ with $\mathcal{N} \models a \in X$, there is $k \in \mathbb{N}$ such that $\mathcal{M} \mid=Y_{k} \subseteq X$.

If $\left\langle Y_{n}: n \in \mathbb{N}\right\rangle$ satisfies the above conditions, we say that it is a describing sequence for $a$ or that it describes a in $\mathcal{N}$ over $\mathcal{M}$.

The existence of cofinal extensions with the description property is almost obvious; indeed, almost the same argument as the one which is used to show that every model has recursively saturated extension establishes the following fact. If $\mathcal{M} \prec \mathcal{N}$ is any cofinal extension of models of Peano Arithmetic, then there exists $\mathcal{K} \succ_{\text {cof }} \mathcal{N}$ such that the extension $\mathcal{M} \prec \mathcal{K}$ has the description property. In particular, every countable recursively saturated model $\mathcal{K}$ of PA has a countable cofinal extension $\mathcal{M}$ with the description property and $\operatorname{SSy}(\mathcal{K}) \varsubsetneqq \operatorname{SSy}(\mathcal{M})$. The following proposition is slightly less obvious.

Proposition 4. Let $\mathcal{M}$ be a countable recursively saturated model of PA. Then there exists $\mathcal{K} \prec_{\text {cof }} \mathcal{M}$ such that the extension has the description property. Moreover we may require $\mathcal{K}$ to be recursively saturated.

Proof. We will prove the first statement first, and then we will show how to modify the argument to get the "moreover" part. It is well known that every recursively saturated model realizes a minimal type (see [13, Chapter 3]). Let $p(x)$ be a minimal type realized in $\mathcal{M}$. Since $p(x)$ is an unbounded type, the set of its realizations is cofinal in $\mathcal{M}$. Let $B$ be a cofinal subset of this set of order type $\omega$. Let $\mathcal{K}$ be the elementary submodel of $\mathcal{M}$ generated by $B$. We will show that the extension $\mathcal{K} \prec \mathcal{M}$ has the description property.

We need to show that $\mathcal{K}$ is a proper submodel of $\mathcal{M}$. This follows, for example, from the fact that $\mathcal{K}$ is not recursively saturated, since it is the union of an $\omega$-chain of minimal elementary end extensions. One can also show 
that in fact $\operatorname{SSy}(\mathcal{K}) \varsubsetneqq \operatorname{SSy}(\mathcal{M})$. Indeed, $\mathcal{M}$ realizes a minimal type that is independent of $p(x)$ (see [13, Chapter 3]). No such type can be realized in $\mathcal{K}$.

Let $u \in \mathcal{M} \backslash \mathcal{K}$ be given. Let $b \in B$ be such that $u<b$. Consider a $\mathcal{K}$-finite set $X$ all of whose elements are below $b$. Since $\mathcal{K}$ is a union of elementary end extensions generated by $B$, there is a Skolem term $t(\bar{x})$ such that $X=t(\bar{b})$, with $\bar{b}=b_{0}, \ldots, b_{r}$. Let $t_{n}(\bar{x})$ be a computable enumeration of all Skolem terms in the variables $\bar{x}$. Let $X_{0}=\left\{x \in \mathcal{K}: x<b_{r}\right\}$, and then recursively define (in $\mathcal{K}) X_{i+1}$ to be $X_{i} \cap t_{i}(\bar{b})$ if $u$ is in this set, and $X_{i} \backslash t_{i}(\bar{b})$ otherwise. Since $\mathcal{M}$ is recursively saturated, the sequence $\left\langle X_{n}: n \in \mathbb{N}\right\rangle$ is coded in $M$. It is clear that it is a describing sequence for $u$ over $\mathcal{K}$.

For the "moreover" part, notice that the whole argument can be repeated starting with a recursively saturated model $(\mathcal{M}, S)$, where $S$ is an inductive partial inductive satisfaction class for $\mathcal{M}$. Then the resulting model $\left(\mathcal{K}, S^{\prime}\right)$ is an elementary submodel of $(\mathcal{M}, S)$. In particular, $S^{\prime}$ is an inductive satisfaction class for $\mathcal{K}$; hence $\mathcal{K}$ is recursively saturated.

We do not know how to improve the above argument to get $\operatorname{SSy}(\mathcal{K})=$ $\operatorname{SSy}(\mathcal{M})$.

In [10] we defined the covering property of cofinal extensions and we proved that covering property implies the automorphism extension property for countable recursively saturated models. Here we will improve it by showing that the description property, which is weaker, also implies the automorphism extension property. This is the main result of this paper.

TheOREM 5. Let $\mathcal{M} \prec_{\text {cof }} \mathcal{R}$ be an extension of models of PA, where both $\mathcal{M}, \mathcal{R}$ are countable and recursively saturated, with the description property. Assume that $g \in \operatorname{Aut}(\mathcal{M})$ and $g^{-1}$ send coded (in $\left.\mathcal{R}\right)$ subsets to coded ones. Then $g$ extends to an automorphism of $\mathcal{R}$.

As usual we shall derive this result from an extension theorem for isomorphisms.

Lemma 6. Let $\mathcal{M}_{1}, \mathcal{R}_{1}, \mathcal{M}_{2}, \mathcal{R}_{2}$ be models of PA such that $\mathcal{M}_{1}$ $\prec_{\text {cof }} \mathcal{R}_{1}, \mathcal{M}_{2} \prec_{\text {cof }} \mathcal{R}_{2}$, and both extensions have the description property. Let $g: \mathcal{M}_{1} \rightarrow \mathcal{M}_{2}$ be an isomorphism such that $g$ sends sets coded in $\mathcal{R}_{1}$ to sets coded in $\mathcal{R}_{2}$ and $g^{-1}$ sends sets coded in $\mathcal{R}_{2}$ to sets coded in $\mathcal{R}_{1}$. Then for every $a \in \mathcal{R}_{1}$ there exists $b \in \mathcal{R}_{2}$ such that there exists an isomorphism $\hat{g}$ : $\operatorname{Hull}^{\mathcal{R}_{1}}\left(\mathcal{M}_{1} \cup\{a\}\right) \rightarrow \operatorname{Hull}^{\mathcal{R}_{2}}\left(\mathcal{M}_{2} \cup\{b\}\right)$ and both extensions $\operatorname{Hull}^{\mathcal{R}_{1}}\left(\mathcal{M}_{1} \cup\{a\}\right) \prec_{\text {cof }} \mathcal{R}_{1}$ and Hull $\mathcal{R}_{2}\left(\mathcal{M}_{2} \cup\{b\}\right) \prec_{\text {cof }} \mathcal{R}_{2}$ have the description property and $\hat{g}$ sends subsets of $\operatorname{Hull}^{\mathcal{R}_{1}}\left(\mathcal{M}_{1}\{a\}\right)$ coded in $\mathcal{R}_{1}$ to subsets of $\operatorname{Hull}^{\mathcal{R}_{2}}\left(\mathcal{M}_{2} \cup\{b\}\right)$ coded in $\mathcal{R}_{2}$.

Of course, this lemma also holds if we exchange the role of $\mathcal{M}_{1}, \mathcal{R}_{1}$ with $\mathcal{M}_{2}, \mathcal{R}_{2}$. The lemma implies the theorem by the standard back-and-forth procedure. We will need one more lemma from [10] (Lemma 3.13 there). 
Lemma 7. If $\mathcal{M} \prec_{\text {cof }} \mathcal{R}$ has the description property, then for every $c \in \mathcal{R}$ the extension $\operatorname{Hull}^{\mathcal{R}}(\mathcal{M}, c) \prec_{\text {cof }} \mathcal{R}$ also has the description property.

Proof of Lemma 6. Let $a \in \mathcal{R}_{1}$ be given. If $a \in \mathcal{M}_{1}$ we let $b=g(a)$ and we are done, so assume that $a \in \mathcal{R}_{1} \backslash \mathcal{M}_{1}$. Pick a sequence $\left\langle X_{n}: n \in \mathbb{N}\right\rangle$, coded in $\mathcal{R}_{1}$, of $\mathcal{M}_{1}$-finite sets describing $a$. Let $Y_{n}=g\left(X_{n}\right)$. This is a sequence of $\mathcal{M}_{2}$-finite sets coded in $\mathcal{R}_{2}$ : indeed, $g$ sends coded sets and sequences to coded ones. Since $g$ is an isomorphism, and, by item 3 in the definition of the description property, for all standard $n, \bigcap_{i \leq n} X_{i} \neq \emptyset$, by overspill in $\mathcal{R}_{2}$ there exists $b$ such that for all $n \in \mathbb{N}, \mathcal{R}_{2} \models b \in Y_{n}$. Pick such a $b$. Thus, by the properties of describing sequences, for every formula $\varphi$ and every $m \in \mathcal{M}_{1}$ we have

$$
\mathcal{R}_{1}=\varphi(m, a) \quad \text { iff } \quad \mathcal{R}_{2}=\varphi(g(m), b) .
$$

Of course, (1) allows one to extend $g$ to $\hat{g}$ in the standard way. Thus, what is needed is to show that $\hat{g}$ sends coded sets to coded ones.

So let $c \in \mathcal{R}_{1}$, so $c$ codes the set $c \cap \operatorname{Hull}^{\mathcal{R}_{1}}\left(\mathcal{M}_{1} \cup\{a\}\right)$. Pick a sequence $\left\langle A_{n}: n \in \mathbb{N}\right\rangle$ describing the pair $\langle a, c\rangle$ over $\mathcal{M}_{1}$, in particular this sequence is coded in $\mathcal{R}_{1}$ and $A_{n} \in \mathcal{M}_{1}$ for standard $n$. We assert that

$$
\forall n \in \mathbb{N} \exists m \in \mathbb{N} \mathcal{M}_{1} \models \forall x \in X_{m} \exists y\langle x, y\rangle \in A_{n} .
$$

Fix $n \in \mathbb{N}$. In order to show $\exists m \mathcal{M}_{1} \models \forall x \in X_{m} \exists y\langle x, y\rangle \in A_{n}$ it suffices to show that there exists $m \in \mathbb{N}$ such that the statement

$$
\forall x \in X_{m} \exists y\langle x, y\rangle \in A_{n}
$$

holds in $\mathcal{R}_{1}$ because $\mathcal{M}_{1} \prec \mathcal{R}_{1}$. In order to see this, it suffices to show that this holds in $\mathcal{R}_{1}$ for all nonstandard $m \in \mathcal{R}_{1}$ (smaller than the length of the sequences coding the sequences used above); granted this one can use underspill. But this statement holds for $x=a$, so it holds in $\mathcal{R}_{1}$ for all $x$ in $X_{m}$ because by the properties of describing sequences if $m$ is nonstandard then all elements of $\mathcal{R}_{1}$ of which $\mathcal{R}_{1}$ thinks they are in $X_{m}$ realize the same type with parameters from $\mathcal{M}_{1}$ in $\mathcal{R}_{1}$.

Granted (2) one argues as follows. Let $B_{n}=g\left(A_{n}\right)$ and $C_{n}=\{y$ : $\left.\langle b, y\rangle \in B_{n}\right\}$. Then $C_{n} \in \mathcal{R}_{2}$, more exactly, $C_{n} \in \operatorname{Hull}^{\mathcal{R}_{2}}\left(\mathcal{M}_{2} \cup\{b\}\right)$. Clearly, the sequence $C_{n}$ describes some $d \in \mathcal{R}_{2}$. Pick such a $d$. Thus, $\langle b, d\rangle$ is in $B_{n}$ for all standard $n$. We assert that $d$ codes $\hat{g} *\left[c \cap \operatorname{Hull}^{\mathcal{R}_{1}}\left(\mathcal{M}_{1} \cup\{a\}\right)\right]$. Let $z \in \operatorname{Hull}^{\mathcal{R}_{1}}\left(\mathcal{M}_{1} \cup\{a\}\right)$. Then $z=t(m, a)$ for some (Skolem) term $t$ and some $m \in \mathcal{M}_{1}$. It follows that $\mathcal{R}_{1} \models t(m, a) \in c$. Thus $\{\langle x, y\rangle$ : $t(m, x) \in y\}$ contains some $A_{n}$, so $\{\langle x, y\rangle: t(g(m), x) \in y\}$ contains $B_{n}$ (with the same $n$ ). Substituting $x=b$ and $y=d$ we see that $d$ contains $t(g(m), b)$ in $\mathcal{R}_{2}$. 
3. Cofinal extensions and large sets. For an element $a$ of a model $\mathcal{M}$ let $\mathcal{M}(a)$ be the smallest elementary initial segment of $\mathcal{M}$ which contains $a$, and let $\mathcal{M}[a]$ be the largest elementary initial segment of $\mathcal{M}$ which does not contain $a$. The gap of $a$, denoted $\operatorname{gap}(a)$, is the set $\mathcal{M}(a) \backslash \mathcal{M}[a]$. The moving gaps lemma states that only the trivial automorphism of a countable recursively saturated model of PA fixes all gaps setwise (see Section 3 in [8] or Section 5 in [11]). The original proof of the lemma uses a type MG(x,y) with the following two properties. (We remark that the proof of the moving gaps lemma given in [13] is different.)

Let $\mathcal{M}=\mathrm{PA}$ be recursively saturated. Then

- For every $a \in \mathcal{M}$ there are arbitrarily large $b \in \mathcal{M}$ such that $(a, b)$ realizes $\mathrm{MG}(x, y)$ in $\mathcal{M}$.

- If $(a, b)$ realizes $\operatorname{MG}(x, y)$ in $\mathcal{M}$, then $a<\operatorname{gap}(b)$, and for every $c \in$ $\operatorname{gap}(b), a \in \operatorname{Hull}(c)$.

From these two properties it follows that if $\mathcal{M} \models \mathrm{PA}$ is recursively saturated and $X \subseteq \mathcal{M}$ has nonempty intersection with all gaps of $\mathcal{M}$, then $\operatorname{Hull}(X)$ $=\mathcal{M}$. Hence we have the following result.

Proposition 8. Let $\mathcal{M}$ be a recursively saturated model of $\mathrm{PA}$, and let $\mathcal{N}$ be its proper cofinal extension. Then there exists $b \in \mathcal{N}$ such that gap ${ }^{\mathcal{N}}(b)$ contains no element of $\mathcal{M}$.

Proof. It is well known that under our assumption, $\mathcal{N}$ is an elementary extension of $\mathcal{M}$ (see Gaifman [3]) and it is recursively saturated (see Smoryński and Stavi [18]). If there were no "new gaps" in $\mathcal{N} \backslash M$, then we could apply the remark preceding the proposition to $X=\mathcal{M}$, which would give a contradiction.

It is interesting that Proposition 8 implies its stronger version in the case when $\mathcal{M}$ is countable.

Proposition 9. If $\mathcal{M} \prec_{\text {cof }} \mathcal{N}$, the extension is proper, and $\mathcal{M}$ is countable and recursively saturated, then there exist $c, d \in \mathcal{N}$ such that $\mathcal{N}(c)<d$ and no element $z$ of $\mathcal{N}$ with $c \leq z \leq d$ is in $\mathcal{M}$.

Proof. Let $S$ be a partial inductive satisfaction class for $\mathcal{M}$ such that $(\mathcal{M}, S)$ is recursively saturated. Let $\bar{S} \subseteq \mathcal{N}$ be such that $(\mathcal{M}, S) \prec(\mathcal{N}, \bar{S})$. Let $\mathcal{K}=(\mathcal{N}, \bar{S})$. Applying Proposition 8 to $(\mathcal{M}, S)$, we get $b \in \mathcal{N}$ such that gap $^{\mathcal{K}}(b)$ contains no elements of $\mathcal{M}$. Now, since $\mathcal{K}(b)$ has a partial inductive satisfaction class $\bar{S}$, it is recursively saturated, and, in particular, it is tall. Hence gap $^{\mathcal{K}}(b)=\mathcal{K}(b) \backslash \mathcal{K}[b]$ must contain $c, d$ such that $\mathcal{N}(c)<d$, and the result follows.

We believe that the assumption that $\mathcal{M}$ is countable is not necessary in the above proposition. In fact, we suspect, but we do not know how to 
prove that if $\mathcal{M}$ is recursively saturated and $\mathcal{N}$ is a cofinal extension of $\mathcal{M}$, then there are no new isolated gaps in $\mathcal{N}$, i.e. there is no $b \in \mathcal{N}$ such that $\operatorname{gap}(b) \cap \mathcal{M}=\emptyset$ and $\mathcal{N}[b]=\sup (\mathcal{M} \cap \mathcal{N}[b])$ and $\mathcal{N}(b)=\inf (M \cap(\mathcal{N} \backslash \mathcal{N}(b))$. But we can prove the following.

Proposition 10. Let $\mathcal{M}$ be a countable model of $\mathrm{PA}$ and let $\mathcal{N}$ be its cofinal extension. If the extension has the description property, then no new gap in $\mathcal{N}$ is isolated.

Proof. Let $b \in \mathcal{N} \backslash \mathcal{M}$ be such that gap ${ }^{\mathcal{N}} \cap \mathcal{M}=\emptyset$. Let $B_{n}$ be a sequence describing $b$ in $\mathcal{N}$ over $\mathcal{M}$. Let $u_{n}=\min \left(B_{n}\right)$ and let $w_{n}=\max \left(B_{n}\right)$. Let $c \in \mathcal{N}$ realize the recursive type consisting of all formulas of the form $\left(t\left(u_{n}\right)<x\right) \wedge(s(x)<b)$, where $s$ and $t$ are Skolem terms in one variable. Then let $d \in \mathcal{N}$ relize the type consisting of the formulas $(t(c)<x) \wedge(s(x)$ $\left.<w_{n}\right)$, where $t$ and $s$ run through all Skolem terms in one variable as well. Then $[c, d] \cap \mathcal{M}=\emptyset$ and $\operatorname{gap}^{\mathcal{N}}(c)<\operatorname{gap}^{\mathcal{N}}(d)$.

For the purposes of this section let us call a subset $X$ of a model $\mathcal{M}$ large if $\operatorname{Hull}(X)=M$. From the remarks preceding Proposition 8 it follows that if $\mathcal{M}$ is recursively saturated, $X \subset \mathcal{M}$, and there is $e \in \mathcal{M}$ such that $X$ meets all gaps above $e$, then $X$ is large. This implies that all definable (with parameters) subsets of a recursively saturated model are large. The assumption that $\mathcal{M}$ is recursively saturated can be eliminated. This follows from the next lemma which is due to Jim Schmerl (see Lemma 2.1.10 in [13]). Reading the lemma and its corollary, keep in mind that "definable" means "definable with parameters."

Lemma 11. Let $\mathcal{M}$ be a model of $\mathrm{PA}$ and let $X$ be a definable unbounded subset of $\mathcal{M}$. Then for every $a \in \mathcal{M}$ there is an unbounded definable $Y \subseteq X$ and a Skolem term $t(x)$ such that for all $x \in Y, t(x)=a$.

COROLlary 12. Every unbounded definable subset of a model of PA is large.

Notice that for any subset $X \subseteq \mathcal{M}$ either $X$ or its complement is large. Suppose none of them is. Let $Y$ be the complement of $X$. Then there is $a$ which is not in the Skolem hull of $X$, and $b$ which is not in the Skolem closure of $Y$. Suppose $a+b$ is in $X$. Since $b$ is not in $\operatorname{Hull}(Y)$, it must be in $X$. Hence, $a=(a+b)-b$ is in $\operatorname{Hull}(X)$, which is a contradiction. If $a+b$ is in $Y$, the argument is similar.

Recall that we call a subset $X$ of a model $\mathcal{M}$ inductive if all induction axioms are satisfied in the structure $(\mathcal{M}, X)$. Every definable set is inductive. We will now show that there are unbounded inductive sets which are not large.

Proposition 13. Let $\mathcal{M}=\mathrm{PA}$ be countable and recursively saturated. Then $\mathcal{M}$ has an unbounded inductive subset which is not large. 
Sketch of proof. Let $p(x)$ be a minimal type realized in $\mathcal{M}$. Let $p(x)=$ $\left\{\varphi_{n}(x): n \in \mathbb{N}\right\}$. Consider the theory $T$ consisting of all induction axioms in the language of PA with an additional unary predicate $E(x)$, together with the sentences

$$
\left\{\forall x\left(E(x) \rightarrow \varphi_{n}(x)\right): n \in \mathbb{N}\right\} \cup\{\forall x \exists y>x E(x)\} .
$$

Clearly, $T$ is consistent. Hence, by resplendency, $\mathcal{M}$ is expandable to a model of $T$. Suppose $(\mathcal{M}, E)$ is a model of $T$. Then $E$ is an unbounded inductive subset of $\mathcal{M}$ and all elements of $E$ realize $p(x)$. It now follows from the well-known properties of minimal types that $\operatorname{Hull}(E)$ must be a proper subset of $\mathcal{M}$. We already mentioned some reasons for it in the proof of Proposition 4. Here is another reason: $p(x)$ is realized in each gap of $\operatorname{Hull}(E)$, and there are gaps of $\mathcal{M}$ which do not realize any minimal types.

Let us say that a subset $X$ of a model $M$ is rather large if the identity is the only automorphism of $M$ which fixes $X$ pointwise. Of course, every large set is rather large. In contrast with the previous proposition we have:

Proposition 14. Every unbounded inductive subset of a recursively saturated countable model of $\mathrm{PA}$ is rather large.

Proof. Let $\mathcal{M}$ be a countable recursively saturated model of PA, and let $X$ be an unbounded inductive subset of $\mathcal{M}$. Consider the structure $\mathcal{M}_{X}=$ $(\mathcal{M}, X)$. By Corollary $12, X$ is a large subset of $\mathcal{M}_{X}$. If $f \in \operatorname{Aut}(\mathcal{M})$ fixes $X$ pointwise, then, in particular, $f \in \operatorname{Aut}\left(\mathcal{M}_{X}\right)$; so now, since $X$ is large in $\mathcal{M}_{X}$ we can conclude that $f=$ id.

4. Fixed point sets. We proved in [8] that an initial segment $I$ of a recursively saturated model $\mathcal{M}$ is a fixed point set of an automorphism of $\mathcal{M}$ if and only if $I$ is strong in $\mathcal{M}$. The proof in [8] is rather involved and some important parts of the argument have been left out. A complete "back-and-forth" proof is given in [12]. Recently, an elegant approach has been developed by Enayat [2]. It allows giving uniform "back-and-forth free" proofs of several known and new results concerning automorphisms and fixed point sets. Applying Enayat's technique we can prove the following generalization of the theorem on extensions of automorphisms to elementary end extensions.

TheOREM 15. Let $\mathcal{M}=\mathrm{PA}$ be countable and recursively saturated, let $I$ be a strong elementary cut of $\mathcal{M}$, and suppose that $f \in \operatorname{Aut}(I)$ is such that $f$ and $f^{-1}$ send coded sets to coded sets. Then there is $g \in \operatorname{Aut}(\mathcal{M})$ such that $f \subseteq g$ and $\operatorname{fix}(f)=\operatorname{fix}(g)$.

Sketch of proof. The complete proof is given in [13, Theorem 8.7.4]. Here is the outline. Because $I$ is strong, it has a partial inductive satisfaction class 
$S$ coded in $\mathcal{M}$. By standard arguments involving minimal types, $(I, S)$ has an elementary end extension $\left(J, S^{\prime}\right)$ which is generated over $I$ by a set of indiscernibles of order type $(\mathbb{Q},<)$. Again by standard arguments, $(J, I) \cong$ $(\mathcal{M}, I)$, in particular $\mathcal{M}$ and $J$ code the same subsets of $I$. By the assumption and the main result of [9], $f$ can be extended to an automorphism $h \in$ $\operatorname{Aut}(J)$. Now, we use the indiscernibles of $\left(J, S^{\prime}\right)$ to construct by a back-andforth argument another automorphism $k \in \operatorname{Aut}\left(J, S^{\prime}\right)$ such that $k(x)=x$ for all $x \in I$, and for all $x \in J \backslash I, k(x) \notin \operatorname{gap}^{\left(J, S^{\prime}\right)}(h(x))$. Consequently, $g=k \circ h$ is an extension of $f$ such that $\operatorname{fix}(f)=\operatorname{fix}(g)$.

We end with a remark concerning possible generalizations of the above theorem to cofinal extensions. One important ingredient is missing: a notion of strongness for cofinal extensions. We have one strong negative result concerning fixed point sets of automorphisms of cofinal extensions:

Proposition 16. Let $\mathcal{M}=\mathrm{PA}$ be countable and recursively saturated. Then $\mathcal{M}$ has a countable cofinal extension such that for all $f \in \operatorname{Aut}(\mathcal{M})$ and all $g \in \operatorname{Aut}(\mathcal{N}), \operatorname{fix}(f) \neq \operatorname{fix}(g)$.

Proof. Let $\mathcal{N}$ be a recursively saturated countable cofinal extension of $\mathcal{M}$ which is not arithmetically saturated (equivalently, $\mathbb{N}$ is not strong in $\mathcal{N}$ ), and such that $\operatorname{SSy}(\mathcal{M})$ is a proper subset of $\operatorname{SSy}(\mathcal{N})$. By a result of [12], for each $g, \operatorname{fix}(g) \cong \mathcal{N}$, and the result follows.

5. Open problems. There are many open problems concerning recursively saturated models of PA, their automorphisms, and their cofinal extensions. Some were mentioned throughout the paper. Here let us just mention two.

Problem 17. Let $\mathcal{M}$ be a countable recursively saturated model of PA. Is there an $\mathcal{N}$ such that $M \prec_{\text {cof }} N, \operatorname{SSy}(\mathcal{M})=\operatorname{SSy}(\mathcal{N})$, and the extension has the description property?

PRoblem 18. Formulate an appropriate notion of strongness for cofinal extensions and prove that if $\mathcal{M} \prec_{\text {cof }} \mathcal{N}$ and $\mathcal{M}$ is strong in $\mathcal{N}$, then there is $g \in \operatorname{Aut}(\mathcal{N})$ such that $\operatorname{fix}(g)=\mathcal{M}$.

If the project described above is successfully carried out, it will open a whole area of study analogous to the well developed theory of extendability of automorphisms to elementary end extensions.

\section{References}

[1] A. Ehrenfeucht, Discernible elements in models of Peano arithmetic, J. Symbolic Logic 38 (1973), 291-292. 
[2] A. Enayat, Automorphisms of models of arithmetic: a unified view, Ann. Pure Appl. Logic 145 (2007), 16-36.

[3] H. Gaifman, A note on models and submodels of arithmetic, in: Conference in Mathematical Logic, London'70, W. Hodges (ed.), Lecture Notes in Math. 255, Springer, 197, 128-144.

[4] —, Models and types of Peano's Arithmetic, Ann. Math. Logic 9 (1976), 223-306.

[5] M. Kaufmann and J. Schmerl, Saturation and simple extensions of models of arithmetic, Ann. Pure Appl. Logic 27 (1984), 109-136.

[6] - - - Remarks on weak notions of saturation in models of Peano Arithmetic, J. Symbolic Logic 52 (1987), 129-148.

[7] R. Kaye, Models of Peano Arithmetic, Oxford Logic Guides 15, Oxford Univ. Press, 1991.

[8] R. Kaye, R. Kossak and H. Kotlarski, Automorphisms of recursively saturated models of arithmetic, Ann. Pure Appl. Logic 55 (1991), 67-91.

[9] R. Kossak and H. Kotlarski, Results on automorphisms of recursively saturated models of PA, Fund. Math. 129 (1988), 9-15.

[10] - - -, On extending automorphisms of models of PA, ibid. 149 (1996), 245-263.

[11] R. Kossak, H. Kotlarski and J. Schmerl, On maximal subgroups of the automorphism group of a countable recursively saturated models of PA, Ann. Pure Appl. Logic 65 (1993), 125-148.

[12] R. Kossak and J. Schmerl, Arithmetically saturated models of arithmetic, Notre Dame J. Formal Logic 36 (1995), 531-546.

[13] - - - The Structure of Models of Peano Arithmetic, Oxford Logic Guides 50, Oxford Univ. Press, 2006.

[14] H. Kotlarski, On elementary cuts in recursively saturated models of arithmetic, Fund. Math. 120 (1984), 205-222.

[15] J. Schmerl, Recursively saturated models generated by indiscernibles, Notre Dame J. Formal Logic 26 (1981), 95-105.

[16] C. Smoryński, Cofinal extensions of nonstandard models of arithmetic, ibid. 22 (1981), 133-144.

[17] -, Elementary extensions of recursively saturated models of arithmetic, ibid., 193203.

[18] C. Smoryński and J. Stavi, Cofinal extensions preserve recursive saturation, in: L. Pacholski et al. (eds.), Model Theory of Algebra and Arithmetic, Lecture Notes in Math. 834, Springer, 1981, 338-345.

The Graduate Center

City University of New York

365 Fifth Avenue

New York, NY 10016, U.S.A.

E-mail: rkossak@gc.cuny.edu
Faculty of Mathematics Cardinal Stefan Wyszyński University Dewajtis 5 01-815 Warszawa, Poland

Received 17 July 2006;

in revised form 31 March 2008 